\title{
Transcultural Adaptation and Validation of the Family Satisfaction in the Intensive Care Unit Questionnaire in a Korean Sample
}

\author{
Youlim Kim 1,2, Jinsoo Min' , Gajin Lim ${ }^{1,2}$, Jung-Kyu Lee ${ }^{2,3}$, Hannah Lee ${ }^{4}$, Jinwoo Lee ${ }^{2}$, Kyung Su Kim ${ }^{5}$, \\ Jong Sun Park ${ }^{1,2}$, Young-Jae Cho ${ }^{1,2}$, You Hwan Jo ${ }^{6}$, Hogeol Rhu ${ }^{4}$, Kyu-seok Kim ${ }^{6}$, Sang-Min Lee ${ }^{2}$, and Yeon Joo Lee ${ }^{1,2}$ \\ ${ }^{1}$ Department of Internal Medicine, Seoul National University Bundang Hospital, Seongnam; ${ }^{2}$ Department of Internal Medicine, Seoul National University College of Medicine, Seoul; \\ ${ }^{3}$ Department of Internal Medicine, Metropolitan Government Seoul National University Boramae Medical Center, Seoul; ${ }^{4}$ Department of Anesthesiology, ${ }^{5}$ Department of Emergency \\ Medicine, Seoul National University College of Medicine, Seoul; ${ }^{6}$ Department of Emergency Medicine, Seoul National University Bundang Hospital, Seongnam, Korea
}

Background: A number of questionnaires designed for analyzing family members' inconvenience and demands in intensive care unit (ICU) care have been developed and validated in North America. The family satisfaction in the intensive care Unit-24 (FS-ICU-24) questionnaire is one of the most widely used of these instruments. This study aimed to translate the FS-ICU-24 questionnaire into Korean and validate the Korean version of the questionnaire.

Methods: The study was conducted in the medical, surgical, and emergency ICUs at three tertiary hospitals. Relatives of all patients hospitalized for at least 48 hours were enrolled for this study participants. The validation process included the measurement of construct validity, internal consistency, and interrater reliability. The questionnaire consists of 24 items divided between two subscales: satisfaction with care (14 items) and satisfaction with decision making (10 items).

Results: In total, 200 family members of 176 patients from three hospitals completed the FS-ICU-24 questionnaire. Construct validity for the questionnaire was superior to that observed for a visual analog scale (Spearman's $r=0.84, p<0.001$ ). Cronbach's as were 0.83 and 0.80 for the satisfaction with care and satisfaction with decision making subscales, respectively. The mean ( \pm standard deviation) total FS-ICU-24 score was $75.44 \pm 17.70$, and participants were most satisfied with consideration of their needs $(82.13 \pm 21.03)$ and least satisfied with the atmosphere in the ICU waiting room (35.38 \pm 34.84$)$.

Conclusions: The Korean version of the FS-ICU-24 questionnaire demonstrated good validity and could be a useful instrument with which to measure family members' satisfaction about ICU care.

Key Words: family; intensive care units; satisfaction; validation studies.

\section{Introduction}

Most intensive care unit (ICU) patients cannot explain their symptoms or complaints, because intubation and other ICU-related procedures prevent them from communicating with their doctors or other caregivers [1]. Therefore, family members often participate in decision making on behalf of patients during the treatment process [2]. However, over half of such family members have often experienced anxiety or depression and those experiences might affect decision making in the ICU [3]. Additionally, improved satisfaction of the ICU patients' relatives appears to reduce ICU admission

\footnotetext{
Received on November 23, 2016 Revised on February 14, 2017 Accepted on February 14, 2017

Correspondence to: Yeon Joo Lee, Department of Internal Medicine, Seoul National University Bundang Hospital, 82 Gumi-ro 173beon-gil, Bundang-gu, Seongnam 13620, Korea Tel: +82-31-787-7082, Fax: +82-31-787-4052, E-mail: yjlee1117@snubh.org
}

${ }^{*}$ No potential conflict of interest relevant to this article was reported.

cc This is an Open Access article distributed under the terms of the Creative Commons Attribution Non-Commercial License (http://creativecommons.org/ licenses/by-nc/4.0/) which permits unrestricted non-commercial use, distribution, and reproduction in any medium, provided the original work is properly cited. Copyright (c) 2017 The Korean Society of Critical Care Medicine 
days and increase the psychological quality of family members $[4,5]$. Therefore, understanding family members' needs and supporting their emotional stress through frequent communication are important for ICU intensivists [1].

Since the 1970s, several questionnaires have been presented to identify the family members' demands and satisfaction with care, with ICU-related [6,7], and the family satisfaction in the intensive care unit-24 (FSICU-24) questionnaire is one of the most widely used instruments. Since Heyland and Tranmer [6] developed the FS-ICU-24 questionnaire in 2001, both the original and modified versions of have been used, and it has been adapted for use in other countries such as Turkey and Germany $[8,9]$.

To translate the FS-ICU-24 questionnaire is fundamental if it is to be used to assess patients' family members' satisfaction with ICUs within a particular culture and perform a multicultural comparison. There is no validation model for use of the FS-ICU-24 questionnaire as for assessing of patients' family members' satisfaction with ICU care in Korea. Therefore, this study aimed to translate the FS-ICU-24 questionnaire into Korean and validate the Korean version of the questionnaire and used patient data to evaluate interrater reliability between patients' family members.

\section{Materials and Methods}

\section{1) Study design and data collection}

The study was conducted prospectively in the medical, surgical, and emergency ICUs at three tertiary hospitals (two are tertiary hospitals with more than 1,000 beds and the third has approximately 700 beds; each hospital has 63, 65, and 32 ICU beds, respectively) between January 2015 and February 2016. Most patients who were admitted to the participating ICUs had diseases such as pulmonary or cardiac problems, but post-surgical patients were also treated in the ICUs. Relatives of all patients hospitalized for at least 48 hours were considered eligible for participation in the study. Patients' relatives included spouses, parents, children, and siblings, and we endeavored to obtain responses from at least two relatives for each patient. Physicians provided the questionnaires to family members and received completed questionnaires immediately or at the next ICU visiting time.

\section{2) FS-ICU-24 questionnaire}

Data were collected using the Korean translation of the FS-ICU-24 questionnaire, which was developed and validated by Heyland and Tranmer [6] in 2001 and modified by Wall et al. [10] in 2007. We obtained permission to use the questionnaire from one of the developers prior to commencement of the study. Two experts translated the original questionnaire into Korean individually, and then integrated their individual translations to form a single translation. This was retranslated into English by two additional experts, and an English language expert compared this version to the original version. The Korean version was considered complete upon confirmation that it did not differ from the original version (the final Korean version of FS-ICU-24 questionnaire was added to the supplementary materials) [11].

The survey was divided into two sections: a visual ana$\log$ scale (VAS) and a 24-item questionnaire. In the first section, patients' family members indicated their overall satisfaction, satisfaction with care, and satisfaction with decision making using the VAS, which ranged from 1 to 10 , with lower scores indicating greater satisfaction. In the second section, patients' family members indicated their satisfaction with ICU care by responding to closed questions in the questionnaire. The 24 questionnaire items were divided between two subscales: satisfaction with care (14 items) and satisfaction with decision making (10 items). Responses were provided using a scale ranging from 1 (excellent) to 5 (very poor), and each item was recoded to provide a score between 0 and 100, as follows: $0=$ very poor, $25=$ poor, $50=$ good, $75=$ very good, and $100=$ excellent [6]. Higher scores indicated greater satisfaction. 


\section{3) Validation and statistical analysis}

Data were analyzed using SPSS software for Windows version 18.0 (SPSS Inc., Chicago, IL, USA). Categorical and continuous data were presented as means and standard deviations, and a chi-square test was performed to compare the categorical variables. In this study, the validation was evaluated by construct validity, internal consistency, and inter-rater reliability. Construct validity was assessed by comparing the VAS scores obtained in the first section of the survey with the scores for the 24 questionnaire items included in the second section of the survey, using Spearman's correlation coefficients. Internal consistency was evaluated using Cronbach's $\alpha$ for each item in the questionnaire. Inter-rater reliability

Table 1. Baseline characteristics of intensive care unit patients

\begin{tabular}{lc}
\hline Variables & Patients \\
\hline Age & $64.46 \pm 17.38$ \\
\hline Sex (male) & $109 / 168(64.9)$ \\
\hline Previous intensive care unit Hx. (yes) & $76 / 168(45.2)$ \\
\hline Length of stay & $14.95 \pm 13.41$ \\
\hline APACHE II score & $23.40 \pm 10.71$ \\
Intensive care unit survivor & $136 / 168(81.0)$ \\
Cause of intensive care unit admission & \\
\hline Respiratory & $70(41.7)$ \\
\hline Cardiovascular & $21(12.5)$ \\
\hline Neurologic & $3(1.8)$ \\
\hline Sepsis, septic shock & $32(19.0)$ \\
\hline Renal replacement therapy & $16(9.5)$ \\
\hline Others & $26(15.5)$ \\
\hline
\end{tabular}

Values are expressed as mean \pm standard deviation or $n(\%)$.

Hx.: history; APACHE II: Acute physiology and chronic health evaluation score. was evaluated by comparing responses between different family members of the same patient using intraclass correlation coefficients (ICCs). The significance level was set at $\mathrm{p}<0.05$.

\section{Results}

\section{1) Patients' and family members' baseline characteristics}

Table 1 and 2 show the baseline characteristics for the ICU patients and their family members. The patients' average age was $64.46 \pm 17.38$ years, and more than half of the patients were men $(n=109,64.9 \%)$. Their mean duration of hospitalization in the ICU was $14.95 \pm 13.41$ days, and their average Acute Physiology and Chronic Health Evaluation-II score was $23.40 \pm 10.71$. The sex distribution of the overall sample was even (male to female ratio $=1: 1$, and most of the patients' relatives were their children or spouses.

\section{2) Construct validity}

Spearman's correlation coefficients for overall satisfaction, satisfaction with care, and satisfaction with decision making were $0.84(\mathrm{p}<0.001), 0.83(\mathrm{p}<0.001)$, and $0.80(\mathrm{p}<0.001)$, respectively (Table 3$)$. In the subgroup analysis of construct validity with the enrolled three hospitals, the results were consistent with those of the total population (Table 3).

\section{3) Internal Consistency}

Cronbach's $\alpha$ for satisfaction with care (0.94) and satis-

Table 2. Baseline characteristics of family members

\begin{tabular}{lccc}
\hline Relationship & Value & Age & Sex (M:F) \\
\hline Offspring & $112(56.0)$ & $46.95 \pm 11.91$ & $59: 53$ \\
Spouse & $50(25.0)$ & $61.14 \pm 11.93$ & $22: 28$ \\
Parents & $13(6.5)$ & $51.42 \pm 12.98$ & $6: 7$ \\
Other family $^{*}$ & $13(6.5)$ & $53.08 \pm 22.39$ & $5: 8$ \\
Siblings & $12(6.0)$ & $52.92 \pm 8.94$ & $8: 4$ \\
\hline
\end{tabular}

Values are expressed as mean \pm standard deviation or $n(\%)$.

M: male; F: female.

*Other family included uncle, aunt, cousins and so on. 
Table 3. Construct validity

\begin{tabular}{|c|c|c|}
\hline Variables & r & p-value \\
\hline \multicolumn{3}{|l|}{ Total group } \\
\hline Overall satisfaction & 0.84 & $<0.001$ \\
\hline Overall satisfaction with care & 0.83 & $<0.001$ \\
\hline Overall satisfaction with information and decision making & 0.80 & $<0.001$ \\
\hline \multicolumn{3}{|l|}{ Subgroup analysis } \\
\hline \multicolumn{3}{|l|}{ Hospital A } \\
\hline Overall satisfaction & 0.87 & 0.002 \\
\hline Overall satisfaction with care & 0.82 & 0.005 \\
\hline Overall satisfaction with information and decision making & 0.84 & 0.006 \\
\hline \multicolumn{3}{|l|}{ Hospital B } \\
\hline Overall satisfaction & 0.84 & $<0.001$ \\
\hline Overall satisfaction with care & 0.84 & $<0.001$ \\
\hline Overall satisfaction with information and decision making & 0.80 & $<0.001$ \\
\hline \multicolumn{3}{|l|}{ Hospital C } \\
\hline Overall satisfaction & 0.77 & $<0.001$ \\
\hline Overall satisfaction with care & 0.76 & $<0.001$ \\
\hline Overall satisfaction with information and decision making & 0.75 & $<0.001$ \\
\hline
\end{tabular}

faction with decision making $(0.88)$ were both high (Table 4). Additionally, all items about both the satisfaction with care and satisfaction with decision making subscales demonstrated high levels of internal consistency regardless of whether other items were deleted. In the subgroup analysis of internal consistency of family satisfaction, Cronbach's alpha scores for each hospital were similar to those of the total group (Table 4).

\section{4) Reliability}

Twenty-four pairs of responses provided by two family members of one patient were used to measure interrater reliability (Table 5, Figure 1). The ICC for the satisfaction with care subscale was slightly low $(0.49, \mathrm{p}=0.006)$, with a higher ICC $(0.64, \mathrm{p}<0.001)$ for the satisfaction with decision making subscale.

\section{Discussion}

The study aimed to translate the FS-ICU-24 questionnaire into Korean and validate the translated Korean version of the questionnaire. The results showed that the Korean version showed good construct validity and internal consistency and would be the useful diagnostic tool for evaluating of the satisfaction of family members', experiencing ICU care.

While previous ICU care has focused on increasing survival rates, ICU intensivists have recently become interested in the qualitative improvement of ICU care by enhancing communication with patients and their family members and evaluating their satisfaction $[12,13]$. Several tools, such as the Critical Care Family Needs Inventory, Critical Care Family Satisfaction Survey, and FS-ICU-24 questionnaire, have been used to evaluate satisfaction with ICU care [14-16]. In addition, the original versions of these surveys have been adapted for use in other cultures, such as those of Turkey and The Netherlands $[8,15]$.

In this study, we validated construct validity, internal consistency, and interrater reliability between family members. Results from Table 3 show that the questionnaire demonstrated excellent construct validity, with respect to overall satisfaction, satisfaction with care, and 
Table 4. Internal consistency of family satisfaction

\begin{tabular}{|c|c|c|}
\hline Items & Mean \pm SD & Cronbach's alpha if item deleted \\
\hline \multicolumn{3}{|l|}{ Total group } \\
\hline Cronbach's alpha for satisfaction with care & & 0.94 \\
\hline Courtesy, respect and compassion toward patient & $77.63 \pm 23.43$ & 0.94 \\
\hline Management of pain & $75.13 \pm 25.74$ & 0.94 \\
\hline Management of breathlessness & $79.25 \pm 24.57$ & 0.94 \\
\hline Management of agitation & $68.00 \pm 30.71$ & 0.94 \\
\hline How well staff considered family needs & $82.13 \pm 21.03$ & 0.94 \\
\hline How well staff provided emotional support toward family & $75.88 \pm 25.24$ & 0.94 \\
\hline Coordination and teamwork by staff & $77.50 \pm 22.83$ & 0.94 \\
\hline Courtesy, respect and compassion toward family & $80.38 \pm 21.54$ & 0.94 \\
\hline Skill and competence of intensive care unit nurses & $80.25 \pm 22.08$ & 0.94 \\
\hline Frequency of communication with intensive care unit nurses & $76.00 \pm 26.51$ & 0.94 \\
\hline Skill and competence of intensive care unit doctors & $78.38 \pm 25.27$ & 0.94 \\
\hline Atmosphere of intensive care unit & $69.63 \pm 27.10$ & 0.94 \\
\hline Atmosphere in the intensive care unit waiting room & $35.38 \pm 34.84$ & 0.95 \\
\hline Overall satisfaction with care & $73.00 \pm 24.22$ & 0.94 \\
\hline Cronbach's alpha for satisfaction with decision making & & 0.88 \\
\hline Frequency of communication & $72.25 \pm 26.85$ & 0.86 \\
\hline Ease of getting information & $81.00 \pm 20.86$ & 0.86 \\
\hline Understanding of Information & $80.63 \pm 21.77$ & 0.85 \\
\hline Honesty of Information & $80.13 \pm 23.55$ & 0.85 \\
\hline Completeness of Information & $80.50 \pm 22.16$ & 0.85 \\
\hline Consistency of Information & $79.00 \pm 23.84$ & 0.86 \\
\hline Included in decision making process & $80.63 \pm 24.87$ & 0.87 \\
\hline Support during decision making process & $76.63 \pm 27.12$ & 0.86 \\
\hline Control over the care of your family member & $73.38 \pm 26.65$ & 0.87 \\
\hline Adequate time to have your concerns and questions & $78.00 \pm 41.53$ & 0.90 \\
\hline \multicolumn{3}{|l|}{ Subgroup analysis } \\
\hline \multicolumn{3}{|l|}{ Hospital A } \\
\hline Cronbach's alpha for satisfaction with care & & 0.93 \\
\hline Courtesy, respect, and compassion toward patient & $66.67 \pm 30.62$ & 0.92 \\
\hline Management of pain & $52.78 \pm 42.29$ & 0.92 \\
\hline Management of breathlessness & $72.22 \pm 26.35$ & 0.92 \\
\hline Management of agitation & $75.00 \pm 17.68$ & 0.92 \\
\hline How well staff considered family needs & $41.67 \pm 43.30$ & 0.92 \\
\hline How well staff provided emotional support toward family & $63.89 \pm 25.35$ & 0.92 \\
\hline Coordination and teamwork by staff & $72.22 \pm 19.54$ & 0.92 \\
\hline Courtesy, respect, and compassion toward family & $77.78 \pm 19.54$ & 0.92 \\
\hline Skill and competence of intensive care unit nurses & $75.00 \pm 21.65$ & 0.92 \\
\hline Frequency of communication with intensive care unit nurses & $50.00 \pm 37.50$ & 0.92 \\
\hline Skill and competence of intensive care unit doctors & $72.22 \pm 23.20$ & 0.92 \\
\hline Atmosphere of intensive care unit & $66.67 \pm 25.00$ & 0.93 \\
\hline
\end{tabular}


Table 4. Continued

\begin{tabular}{|c|c|c|}
\hline Items & Mean \pm SD & Cronbach's alpha if item deleted \\
\hline Atmosphere in the intensive care unit waiting room & $36.11 \pm 45.26$ & 0.92 \\
\hline Overall satisfaction with care & $66.67 \pm 21.65$ & 0.92 \\
\hline Cronbach's alpha for satisfaction with decision making & & 0.76 \\
\hline Frequency of communication & $66.67 \pm 21.65$ & 0.75 \\
\hline Ease of getting information & $75.00 \pm 12.50$ & 0.74 \\
\hline Understanding of information & $75.00 \pm 21.65$ & 0.73 \\
\hline Honesty of information & $63.89 \pm 30.90$ & 0.69 \\
\hline Completeness of information & $72.22 \pm 26.35$ & 0.70 \\
\hline Consistency of information & $61.11 \pm 35.60$ & 0.69 \\
\hline Included in decision making process & $72.22 \pm 23.20$ & 0.71 \\
\hline Support during decision making process & $61.11 \pm 25.35$ & 0.70 \\
\hline Control over the care of your family member & $58.33 \pm 33.07$ & 0.72 \\
\hline Adequate time to have your concerns and questions addressed & $55.56 \pm 52.71$ & 0.87 \\
\hline \multicolumn{3}{|l|}{ Hospital B } \\
\hline Cronbach's alpha for satisfaction with care & & 0.95 \\
\hline Courtesy, respect, and compassion toward patient & $74.00 \pm 25.15$ & 0.95 \\
\hline Management of pain & $72.33 \pm 27.75$ & 0.95 \\
\hline Management of breathlessness & $78.33 \pm 25.11$ & 0.95 \\
\hline Management of agitation & $66.33 \pm 30.06$ & 0.95 \\
\hline How well staff considered family needs & $79.33 \pm 23.74$ & 0.95 \\
\hline How well staff provided emotional support toward family & $75.67 \pm 24.65$ & 0.95 \\
\hline Coordination and teamwork by staff & $76.00 \pm 24.47$ & 0.95 \\
\hline Courtesy, respect, and compassion toward family & $78.33 \pm 24.08$ & 0.95 \\
\hline Skill and competence of intensive care unit nurses & $78.33 \pm 23.37$ & 0.95 \\
\hline Frequency of communication with intensive care unit nurses & $73.33 \pm 27.06$ & 0.95 \\
\hline Skill and competence of intensive care unit doctors & $78.00 \pm 25.32$ & 0.95 \\
\hline Atmosphere of intensive care unit & $66.67 \pm 28.57$ & 0.95 \\
\hline Atmosphere in the intensive care unit waiting room & $35.33 \pm 34.41$ & 0.95 \\
\hline Overall satisfaction with care & $74.33 \pm 24.31$ & 0.95 \\
\hline Cronbach's alpha for satisfaction with decision making & & 0.89 \\
\hline Frequency of communication & $69.67 \pm 30.56$ & 0.88 \\
\hline Ease of getting information & $81.33 \pm 20.17$ & 0.88 \\
\hline Understanding of information & $79.33 \pm 21.50$ & 0.88 \\
\hline Honesty of information & $79.00 \pm 24.67$ & 0.88 \\
\hline Completeness of information & $76.67 \pm 23.37$ & 0.88 \\
\hline Consistency of information & $79.00 \pm 23.62$ & 0.88 \\
\hline Included in decision making process & $77.33 \pm 28.82$ & 0.89 \\
\hline Support during decision making process & $71.33 \pm 30.40$ & 0.88 \\
\hline Control over the care of your family member & $69.67 \pm 30.00$ & 0.88 \\
\hline Adequate time to have your concerns and questions addressed & $70.67 \pm 45.84$ & 0.90 \\
\hline
\end{tabular}


Table 4. Continued

\begin{tabular}{|c|c|c|}
\hline Items & Mean \pm SD & Cronbach's alpha if item deleted \\
\hline \multicolumn{3}{|l|}{ Hospital C } \\
\hline Cronbach's alpha for satisfaction with care & & 0.94 \\
\hline Courtesy, respect, and compassion toward patient & $79.35 \pm 22.12$ & 0.94 \\
\hline Management of pain & $77.72 \pm 22.07$ & 0.93 \\
\hline Management of breathlessness & $79.89 \pm 23.22$ & 0.93 \\
\hline Management of agitation & $69.29 \pm 30.81$ & 0.94 \\
\hline How well staff considered family needs & $83.97 \pm 19.11$ & 0.94 \\
\hline How well staff provided emotional support toward family & $75.82 \pm 26.84$ & 0.93 \\
\hline Coordination and teamwork by staff & $77.72 \pm 22.38$ & 0.93 \\
\hline Courtesy, respect and compassion toward family & $81.52 \pm 19.91$ & 0.93 \\
\hline Skill and competence of intensive care unit nurses & $82.61 \pm 19.87$ & 0.93 \\
\hline Frequency of communication with intensive care unit nurses & $80.71 \pm 21.96$ & 0.94 \\
\hline Skill and competence of intensive care unit doctors & $80.71 \pm 24.33$ & 0.93 \\
\hline Atmosphere of intensive care unit & $72.83 \pm 25.32$ & 0.94 \\
\hline Atmosphere in the intensive care unit waiting room & $35.60 \pm 34.62$ & 0.95 \\
\hline Overall satisfaction with care & $72.55 \pm 23.75$ & 0.94 \\
\hline Cronbach's alpha for satisfaction with decision making & & 0.85 \\
\hline Frequency of communication & $75.27 \pm 23.87$ & 0.83 \\
\hline Ease of getting information & $82.88 \pm 18.51$ & 0.83 \\
\hline Understanding of information & $83.15 \pm 20.00$ & 0.82 \\
\hline Honesty of information & $83.42 \pm 18.63$ & 0.82 \\
\hline Completeness of information & $84.24 \pm 19.51$ & 0.83 \\
\hline Consistency of information & $81.25 \pm 21.49$ & 0.83 \\
\hline Included in decision making process & $85.05 \pm 19.63$ & 0.85 \\
\hline Support during decision making process & $82.88 \pm 20.61$ & 0.84 \\
\hline Control over the care of your family member & $77.99 \pm 21.56$ & 0.85 \\
\hline
\end{tabular}

Values are expressed as mean \pm standard deviation (SD). Interpretation of scores: excellent $=100$; very good = 75; good = 50; poor = 25, and very poor $=0$; yes $=100$, no $=0$.

Table 5. Interrater reliability between family members

\begin{tabular}{llr}
\hline Variables & ICCs & p-value \\
\hline Overall satisfaction with care & 0.49 & 0.006 \\
Overall satisfaction with information and decision making & 0.64 & $<0.001$ \\
\hline
\end{tabular}

ICCs, intra-class correlation coefficients.

satisfaction with decision making. A study conducted by Stricker et al. [9], which involved cross-cultural adaptation of the FS-ICU-24 questionnaire, showed that the scale demonstrated construct validity for satisfaction with both care and decision making, but the Spearman's correlation coefficients were lower than those observed in this study [9]. As shown in Table 5, the internal consistency (Cronbach's $\alpha$ ) observed in our study was relatively high, indicating that the Korean version of the scale demonstrated good reliability. In a study of the Turkish version of the scale [8], the internal consistency for each item in the questionnaire ranged from 0.33 to 0.82 . Regarding 

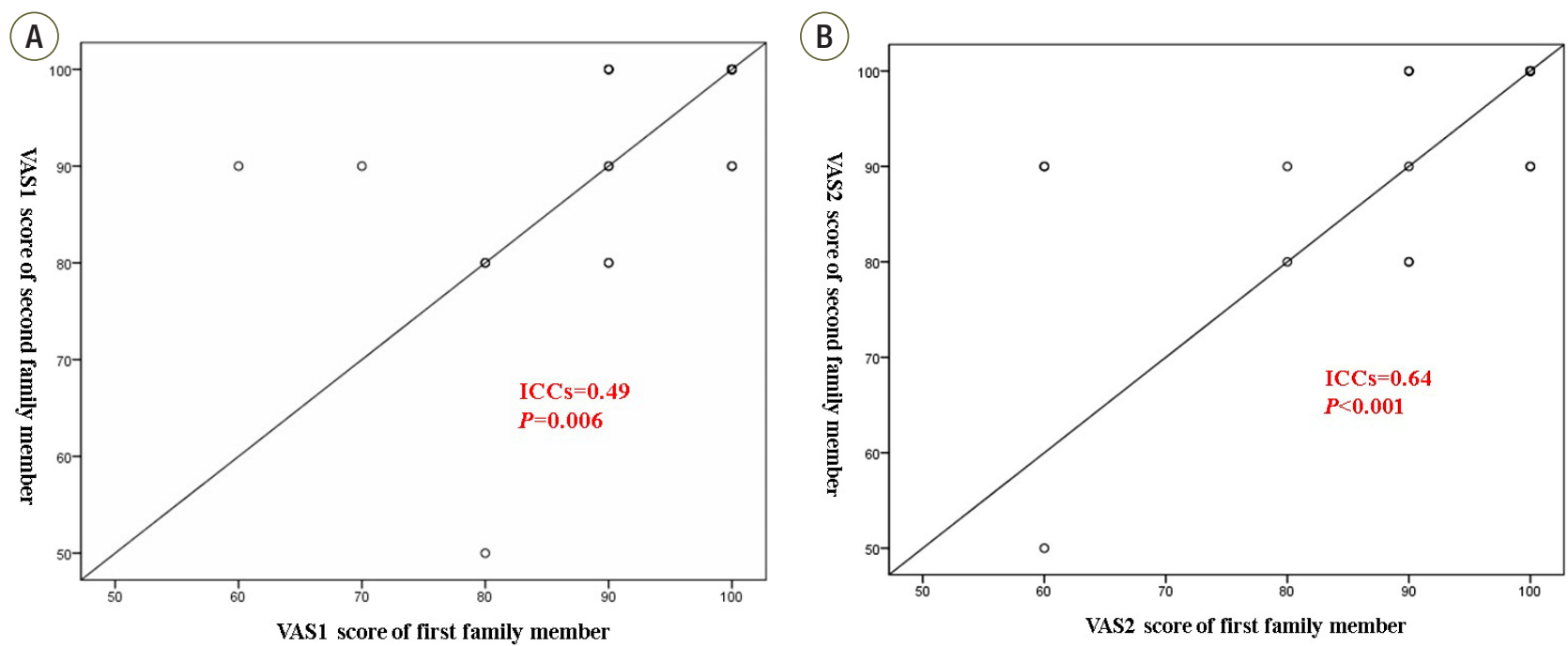

Figure 1. (A) Reliability of satisfaction with care. (B) Reliability of satisfaction with decision making. VAS: visual analog scale; ICC: intraclass correlation coefficient.

agreement in satisfaction between family members, we examined interrater reliability for 24 pairs of responses provided by two relatives of one ICU patient. In our study, while the level of agreement for satisfaction with decision making was adequate, this was not the case for satisfaction with care. A prior cultural adaptation study assessed both interrater and intrarater reliability and showed moderate agreement for satisfaction with care and fair agreement for satisfaction with decision making [9]. However, in our study, as the number of pairs of responses was relatively low and some family members provided disparate responses, this subscale analysis was not possible.

The average score of all items in our study was 75.44 \pm 17.70 , and the mean scores for most items in the satisfaction with care and satisfaction with decision making subscales, with the exception of the "management of agitation," "ICU atmosphere," and "ICU waiting-room atmosphere" items, ranged from 75 to 85 , and the score for the "ICU waiting-room atmosphere" item was lower than that for the other items. In contrast, in the Turkish version, the score for the "communication between nurses and family members" item was lower than those for the other items, which were above the average value of 0.70 [8].

The study was subject to some limitations. For in- stance, responses were provided mainly by relatives of ICU survivors; therefore, the results could have been subject to selection bias. In addition, we used correlation between questionnaire and VAS scores to determine construct validity; however, the VAS was originally designed to measure quality of life or pain $[17,18]$, and might not be an appropriate index for the measurement of satisfaction with care or decision making in family members of ICU patients. Nevertheless, considering that there is no gold standard for the assessment of satisfaction with ICU care, the VAS is one of the most useful instruments for use in assessing and comparing degrees of satisfaction and has been used in similar studies [17-19]. Moreover, as mentioned above, we evaluated inter-rater reliability, and the level of agreement for satisfaction with care was low. In addition, the number of pairs of responses was low and some participants' responses were disparate; therefore, the ICC for satisfaction with overall care was also low. Furthermore, intra-rater reliability was not measured in the study.

In this study, the results indicated that the adapted version of the FS-ICU-24 questionnaire was valid and suitable for use with a Korean sample. Therefore, it could be a valuable tool for use by medical staff in assessing the family members' satisfaction about ICU care in Korea. 


\section{Acknowledgements}

This prospective survey study was conducted in accordance with the amended Declaration of Helsinki. The Institutional Review Board of Seoul National University Bundang Hospital approved the study protocol (IRB No. B-1410-272-005) and all participants provided written informed consent.

We appreciate the efforts of Yong Suk Jo, Joohae Kim, and Ha Youn Lee in the preparation of the manuscript. We also thank the Division of Statistics in Medical Research Collaborating Center at Seoul National University Bundang Hospital for their assistance with the statistical analysis.

\section{ORCID}

Youlim Kim http://orcid.org/0000-0002-1051-0667

Jinsoo Min http://orcid.org/0000-0001-6091-518X

Jong Sun Park http://orcid.org/0000-0003-3707-3636

Young-Jae Cho http://orcid.org/0000-0001-6943-4462

You Hwan Jo http://orcid.org/0000-0002-9507-7603

\section{Supplementary Materials}

The online-only Supplement data is available with this article online: https://doi.org/10.4266/kjccm.2016.00962.

\section{References}

1. Kryworuchko J, Heyland DK. Using family satisfaction data to improve the processes of care in ICU. Intensive Care Med 2009; 35: 2015-7.

2. Davidson JE, Powers K, Hedayat KM, Tieszen M, Kon AA, Shepard E, et al. Clinical practice guidelines for support of the family in the patient-centered intensive care unit: American College of Critical Care Medicine Task Force 2004-2005. Crit Care Med 2007; 35: 605-22.
3. Pochard F, Azoulay E, Chevret S, Lemaire F, Hubert P, Canoui P, et al. Symptoms of anxiety and depression in family members of intensive care unit patients: ethical hypothesis regarding decision-making capacity. Crit Care Med 2001; 29: 1893-7.

4. Lautrette A, Darmon M, Megarbane B, Joly LM, Chevret S, Adrie C, et al. A communication strategy and brochure for relatives of patients dying in the ICU. N Engl J Med 2007; 356: 469-78.

5. Norton SA, Hogan LA, Holloway RG, TemkinGreener H, Buckley MJ, Quill TE. Proactive palliative care in the medical intensive care unit: effects on length of stay for selected high-risk patients. Crit Care Med 2007; 35: 1530-5.

6. Heyland DK, Tranmer JE; Kingston General Hospital ICU Research Working Group. Measuring family satisfaction with care in the intensive care unit: the development of a questionnaire and preliminary results. J Crit Care 2001; 16: 142-9.

7. Wasser T, Matchett S. Final version of the critical care family satisfaction survey questionnaire. Crit Care Med 2001; 29: 1654-5.

8. Tastan S, Iyigun E, Ayhan H, Kilickaya O, Y1lmaz AA, Kurt E. Validity and reliability of Turkish version of family satisfaction in the intensive care unit. Int J Nurs Pract 2014; 20: 320-6.

9. Stricker KH, Niemann S, Bugnon S, Wurz J, Rohrer O, Rothen HU. Family satisfaction in the intensive care unit: cross-cultural adaptation of a questionnaire. J Crit Care 2007; 22: 204-11.

10. Wall RJ, Engelberg RA, Downey L, Heyland DK, Curtis JR. Refinement, scoring, and validation of the family satisfaction in the intensive care unit (FS-ICU) survey. Crit Care Med 2007; 35: 271-9.

11. Beaton DE, Bombardier C, Guillemin F, Ferraz MB. Guidelines for the process of cross-cultural adaptation of self-report measures. Spine (Phila Pa 1976) 2000; 25: 3186-91.

12. Hall RI, Rocker GM. End-of-life care in the ICU: treatments provided when life support was or was not withdrawn. Chest 2000; 118: 1424-30. 
13. Baker R, Wu AW, Teno JM, Kreling B, Damiano AM, Rubin HR, et al. Family satisfaction with endof-life care in seriously ill hospitalized adults. J Am Geriatr Soc 2000; 48(5 Suppl): S61-9.

14. Henrich NJ, Dodek P, Heyland D, Cook D, Rocker G, Kutsogiannis D, et al. Qualitative analysis of an intensive care unit family satisfaction survey. Crit Care Med 2011; 39: 1000-5.

15. Bijttebier P, Delva D, Vanoost S, Bobbaers H, Lauwers $\mathrm{P}$, Vertommen H. Reliability and validity of the critical care family needs inventory in a Dutch-speaking Belgian sample. Heart Lung 2000; 29: 278-86.

16. Hickman RL Jr, Daly BJ, Douglas SL, Burant CJ. Evaluating the critical care family satisfaction survey for chronic critical illness. West J Nurs Res 2012; 34: 377-95.

17. EuroQol Group. Euroqol--a new facility for the measurement of health-related quality-of-life. Health Policy 1990; 16: 199-208.

18. Spitzer WO, Dobson AJ, Hall J, Chesterman E, Levi J, Shepherd R, et al. Measuring the quality of life of cancer patients: a concise QL-index for use by physicians. J Chronic Dis 1981; 34: 585-97.

19. Brokelman RB, Haverkamp D, van Loon C, Hol A, van Kampen A, Veth R. The validation of the visual analogue scale for patient satisfaction after total hip arthroplasty. Eur Orthop Traumatol 2012; 3: 101-5. 